\title{
Threat-anticipatory psychophysiological response is enhanced in youth with anxiety disorders and correlates with prefrontal cortex neuroanatomy
}

\author{
Rany Abend, PhD; Mira A. Bajaj, BA; Anita Harrewijn, PhD; Chika Matsumoto, BS; \\ Kalina J. Michalska, PhD; Elizabeth Necka, PhD; Esther E. Palacios-Barrios, MS; \\ Ellen Leibenluft, MD; Lauren Y. Atlas, PhD; Daniel S. Pine, MD
}

\begin{abstract}
Background: Threat anticipation engages neural circuitry that has evolved to promote defensive behaviours; perturbations in this circuitry could generate excessive threat-anticipation response, a key characteristic of pathological anxiety. Research into such mechanisms in youth faces ethical and practical limitations. Here, we use thermal stimulation to elicit pain-anticipatory psychophysiological response and map its correlates to brain structure among youth with anxiety and healthy youth. Methods: Youth with anxiety $(n=25)$ and healthy youth $(n=25)$ completed an instructed threat-anticipation task in which cues predicted nonpainful or painful thermal stimulation; we indexed psychophysiological response during the anticipation and experience of pain using skin conductance response. High-resolution brain-structure imaging data collected in another visit were available for 41 participants. Analyses tested whether the 2 groups differed in their psychophysiological cue-based pain-anticipatory and pain-experience responses. Analyses then mapped psychophysiological response magnitude to brain structure. Results: Youth with anxiety showed enhanced psychophysiological response specifically during anticipation of painful stimulation $(b=0.52, p=0.003)$. Across the sample, the magnitude of psychophysiological anticipatory response correlated negatively with the thickness of the dorsolateral prefrontal cortex $\left(p_{F W E}<0.05\right)$; psychophysiological response to the thermal stimulation correlated positively with the thickness of the posterior insula $\left(p_{F W E}<0.05\right)$. Limitations: Limitations included the modest sample size and the cross-sectional design. Conclusion: These findings show that threat-anticipatory psychophysiological response differentiates youth with anxiety from healthy youth, and they link brain structure to psychophysiological response during pain anticipation and experience. A focus on threat anticipation in research on anxiety could delineate relevant neural circuitry.
\end{abstract}

\section{Introduction}

Anticipation of threat engages conserved neural circuitry that has evolved to facilitate defensive behaviours; ${ }^{1-3}$ perturbations in this circuitry could generate excessive anticipatory response, a core element of pathological anxiety. ${ }^{4,5}$ Grounding research on mechanisms of perturbed threat anticipation in youth is important, because individual differences in threat anticipation and anxiety arise early. ${ }^{6,7}$ However, ethical and practical issues limit such work. In this study, we applied painful thermal stimulation to elicit anticipatory psychophysiological response in youth with anxiety and healthy youth. We tested whether painanticipatory and pain-experience response differentiated patients with anxiety from healthy youth, and mapped response magnitude to brain structure.
Threat anticipation facilitates defensive response, ${ }^{1-3,8}$ and can be indexed in humans using skin conductance response (SCR). ${ }^{5,9,10}$ When anticipatory response is excessive, it can be maladaptive and contribute to anticipatory fears, worry and avoidance - core features of pathological anxiety. ${ }^{4,11}$ Indeed, relative to healthy participants, patients with anxiety show greater psychophysiological response in threat-anticipation conditions, such as those that occur in threat-learning and unpredictable-threat paradigms. ${ }^{4,6,12,13}$ However, the neural mechanisms that generate excessive anticipatory psychophysiological responses in anxiety remain incompletely specified.

Anxiety disorders typically emerge in late childhood or adolescence, ${ }^{14,15}$ heightening the importance of research into aberrant threat-anticipatory response in youth. Relative to adults with anxiety, children and adolescents with anxiety

Correspondence to: R. Abend, Emotion and Development Branch, National Institute of Mental Health, National Institutes of Health, 9000 Rockville Pike, Bldg 15K, MSC-2670, Bethesda, MD; rany.abend@ nih.gov

Submitted June 9, 2020; Revised Aug. 27, 2020; Accepted Sept. 14, 2020

DOI: $10.1503 /$ jpn.200110 
are less likely to have additional disorders or long-term treatment exposure. ${ }^{7}$ Therefore, studies in youth can examine circuitry early in the course of potentially chronic disorders, before comorbidity, treatment and other factors can alter circuitry with development. Psychophysiological and neural correlates of anxiety-related aberrant anticipatory response that are identified in childhood could then provide information about the pathogenesis of anxiety and might be used as biomarkers. ${ }^{5,14,16,17}$

Functional imaging research in adults examines the anticipation of aversive stimuli. Some studies have used potent stimuli such as physical pain to robustly elicit anticipatory states. This work has implicated networks such as the insular and prefrontal cortices, as well as connected limbic structures. ${ }^{4,8,10,18-21}$ Such studies have primarily contrasted neural function during anticipation with safety conditions; relatively few studies have directly linked brain function to anticipatory psychophysiological response (e.g., Seifert and colleagues, ${ }^{10}$ Wendt and colleagues ${ }^{22}$ and $\mathrm{Gu}$ and colleagues ${ }^{23}$ ), presenting a gap in the literature.

A second gap is the limited focus on youth in such research, because of ethical and practical concerns. ${ }^{24}$ Specifically, the MRI environment is inherently anxiogenic because of participant confinement and the production of loud sounds, and participant monitoring is done remotely. ${ }^{25}$ In pediatric patients with anxiety disorders, this complicates imaging research that involves exposure to additional aversive stimuli, which may become intolerable. ${ }^{24}$ Studies aiming to induce fear in youth with anxiety have largely been limited to the use of static emotional faces, ${ }^{17,26}$ which show only low reliability in eliciting neural responses (unpublished observations) and evoke only low levels of fear. ${ }^{27}$ Thus, despite the importance of examining neural correlates of threat-anticipation responses in youth with anxiety, the use of potent aversive stimuli in MRI environments has been avoided in such research.

Structural imaging might address some of these gaps and extend findings from functional studies on threat anticipation in adults. Data on responses to potent aversive stimuli can be acquired separately from scanning, while youth are closely observed in a setting that lacks the confining features of the scanner. Brain-structure data acquired separately could then be used to examine associations between the psychophysiology and neuroanatomy of threat anticipation. Although brain structure variably maps onto function, ${ }^{28}$ results from previous studies in adult humans and rodents do show overlap in the structural and functional correlates of acquired anticipatory psychophysiological response. ${ }^{29-33}$

Our previous work used Pavlovian threat learning across a wide age range to begin exploring the relations between brain structure and threat-anticipatory response, using an aversive visuo-auditory stimulus. ${ }^{34}$ We found that, relative to healthy youth, youth and adults with anxiety disorders showed greater anticipatory psychophysiological response, but comparable response to the unconditioned aversive stimulus itself. Enhanced anticipatory response was further associated with a thinner dorsolateral prefrontal cortex (dlPFC) - a region implicated in regulatory function. ${ }^{18,35,36}$
This finding may suggest that the dlPFC structure could be involved in the regulation of threat-anticipation response.

In the present study, we have extended this work in $2 \mathrm{im}-$ portant ways. First, in our previous study, participants rapidly habituated in their response to the visuo-auditory stimulus, creating a need for more potent stimuli. ${ }^{24}$ In the present study, we applied painful thermal stimulation, which robustly and consistently engages conserved defensive circuitry. ${ }^{37,38}$ Second, we previously induced threat anticipation through uninstructed, probabilistic Pavlovian learning; accordingly, threat occurrence was uncertain and anxietyrelated response was enhanced to both threat and safety cues, complicating inferences about threat-specific anticipation effects. ${ }^{4,39}$ In the present study, we used a task in which cue relation to painful (threat) versus nonpainful (safety) stimulation was instructed, to induce larger differentiations in threat versus safety-cue response and facilitate tests of the specificity of anticipatory response to threat. ${ }^{4,39}$

This is the first study to characterize associations between brain structure and psychophysiological pain-anticipatory response in healthy youth and youth with anxiety. Twenty-five medication-free, treatment-seeking pediatric patients with anxiety and 25 healthy youth completed an instructed threatanticipation task. ${ }^{40,41}$ Cues predicted painful or nonpainful thermal stimulation, and we used SCR to index psychophysiological responses during pain anticipation and pain experience. We conducted brain-structure imaging in a separate visit. In our primary analyses, we examined cue-based painanticipatory SCR and its brain-structure correlates. We hypothesized that patients would show greater anticipatory SCR than healthy youth, specifically to threat cues, and that reduced dlPFC thickness would be associated with greater SCR. ${ }^{34}$ As well, to extend our previous findings on the specificity of anxiety effects to threat anticipation, ${ }^{34}$ we examined psychophysiological response to pain stimulation and its brain-structure correlates. ${ }^{8,17,42}$ Given our previous findings, ${ }^{34}$ we hypothesized that patients with anxiety and healthy youth would show comparable SCR to pain; we hypothesized that SCR to heat pain would be correlated with structure in brain regions previously implicated in response to pain - including the insula, somatosensory cortex and thalamus ${ }^{10,43,44}$ — but that these associations would not be moderated by anxiety.

\section{Methods}

\section{Participants}

Participants were 64 youth recruited from the community for research at the National Institute of Mental Health, including 33 treatment-seeking patients and 31 healthy youth. Data were excluded for participants who did not reliably rate pain during calibration (see below; 2 healthy participants, 2 patients with anxiety); aborted the task (1 healthy participant, 5 patients with anxiety); or technical problems ( 2 healthy participants, 1 patient with anxiety). The final sample included 50 participants. Patients completed the study before treatment. Written informed consent was obtained from the parents of participants; written assent was 
obtained from youth. Procedures were approved by the National Institute of Mental Health Institutional Review Board. Participants received monetary compensation.

\section{Anxiety diagnosis and symptom severity}

All participants were interviewed by trained clinicians using the Kiddie Schedule for Affective Disorders and Schizophrenia for School-Age Children-Present and Lifetime Version (KSADS-PL) ${ }^{45}$ Exclusionary criteria included an intelligence quotient (IQ) less than 70;4 a diagnosis of autism spectrum disorder, posttraumatic stress disorder, schizophrenia, attention-deficit/hyperactivity disorder, obsessive-compulsive disorder, neurologic disorder or major depressive disorder; recent use of psychoactive substances; history of trauma; or significant medical illness. Patients with anxiety were required to meet criteria for generalized, social or separation anxiety disorder. Healthy participants did not meet the criteria for any psychiatric diagnosis. To assess the severity of current anxiety symptoms, we administered the child- and parent-report Screen for Child Anxiety Related Emotional Disorders (SCARED) ${ }^{47}$; see Appendix 1, available at jpn.ca/200110-a1.

\section{Thermal stimulation}

Thermal stimulation was delivered to the left forearm using a CHEPS thermode (27 mm diameter; Medoc Ltd.) for $5 \mathrm{~s}$ per trial (0.5 s ramp-up, $4 \mathrm{~s}$ at target temperature and $0.5 \mathrm{~s}$ ramp-down).

Participants first completed an adaptive staircase pain calibration $^{40,41}$ (Appendix 1). Briefly, participants provided verbal pain ratings (0-10) to varying delivered temperatures. We used individual ratings to estimate temperatures that would reliably elicit subjective pain ratings of 2 (low pain), 5 (mod- erate pain) and 8 (high pain). Participants were required to show a reliable temperature-ratings correlation $\left(R^{2} \geq 0.40\right)^{40,41}$ to start the task. Groups did not differ in terms of calibration indices (Appendix 1).

\section{Threat-anticipation task}

The threat-anticipation task consisted of visual stimuli (yellow triangle, blue square) paired with thermal stimulation. Participants were instructed that 1 shape predicted low-pain stimulation (corresponding to a level 2 pain rating) and the other predicted highly painful stimulation (level 8 pain); we counterbalanced the shape-pain assignment across participants.

In each trial (Figure 1), participants were presented with the low- or high-pain cue (2 s), and then 6 seconds after cue offset, participants received the thermal stimulation (low-, moderate- or high-pain temperature; $5 \mathrm{~s}$ ). After a variable delay (5-7 s), participants used a mouse to provide their pain ratings on a scale of 0 to 10 . Variable intertrial intervals (4-6s) separated the trials.

The task comprised 68 trials, which included combinations of a low-pain cue followed by a low-pain temperature (LL) and a high-pain cue followed by a high-pain temperature $(\mathrm{HH})$. We also included trials in which low- and high-pain cues were followed by a moderate-pain temperature (level 5 pain; LM, HM), to examine anticipatory effects on pain experience. Trials were delivered in 8 blocks in a set order (Appendix 1, Table S1). Blocks included either LL and HH combinations or LM and HM combinations; within blocks, trial order was randomized. At the start of each block, participants provided pain-expectation ratings and the experimenter moved the thermode to a different arm location.

Following the conclusion of block 4, participants were instructed that the initial contingencies had reversed. This

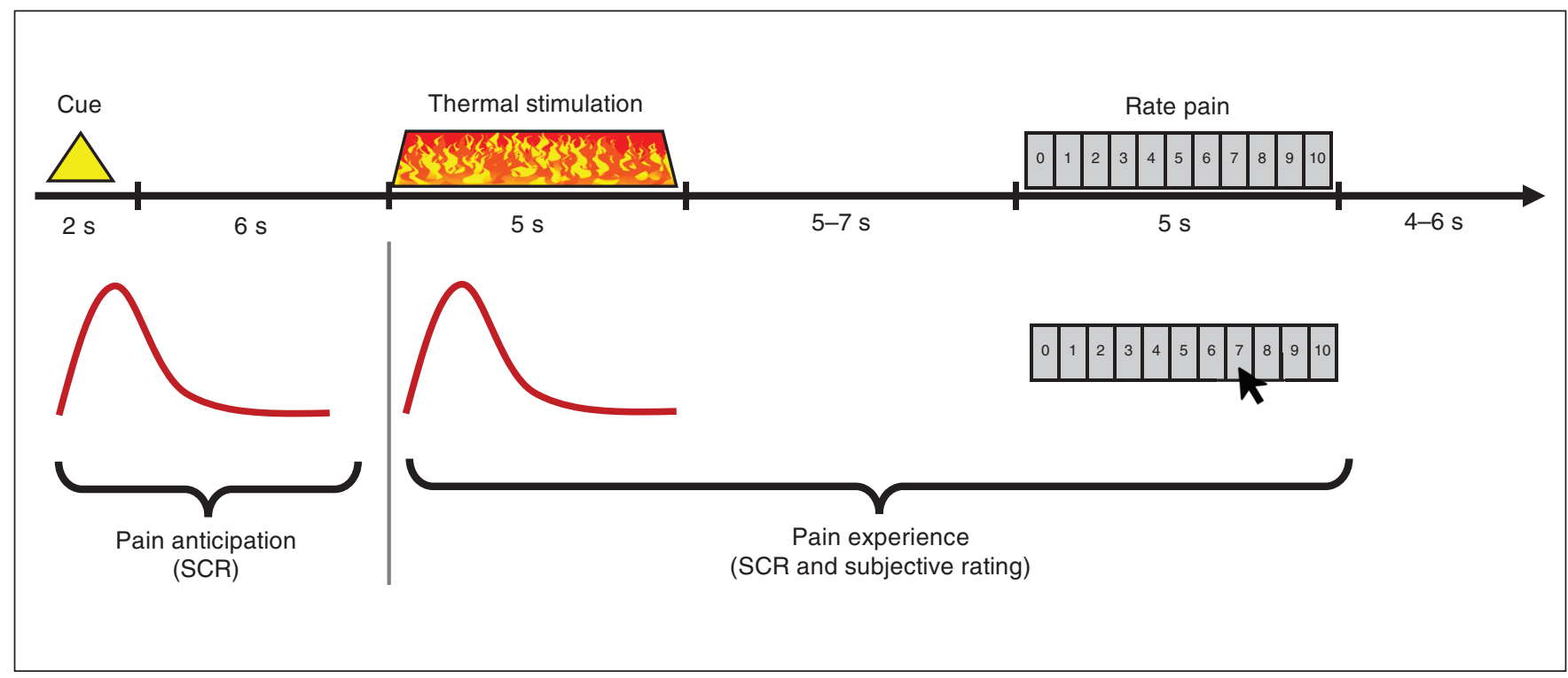

Fig. 1: Threat-anticipation task. Timeline of a single trial in the task (top) and corresponding measures of interest used in the analyses (bottom), which included SCR to index cue-based pain anticipation and SCR and subjective pain ratings to index responses to experienced pain. $\mathrm{SCR}=$ skin conductance response. 
reversal phase was exploratory; analyses of this phase are reported in Appendix 1.

\section{Psychophysiological recording and processing}

We used SCR data to assess psychophysiological responses. The SCR data were derived from skin-conductance data recorded continuously at $1000 \mathrm{~Hz}$ from the left middle and index fingers using Biopac equipment and AcqKnowledge software. We used continuous deconvolution analysis (Ledalab, MATLAB package ${ }^{48}$ ) to decompose skin-conductance data into tonic and phasic components, ${ }^{49}$ as in previous work. ${ }^{50}$ We analyzed data within a $7 \mathrm{~s}$ window, starting with stimulus onset (cue or heat). We conducted analyses on the square root $^{9}$ of the average phasic component of the SCR in the window. ${ }^{49}$ Analyses used multilevel linear mixed-effects regression models (lme4 function in $\mathrm{R}^{51}$; Satterthwaite approximation of degrees of freedom), with participant intercept as a random effect. We considered trial data at the individual participant level to calculate $\beta$ values for within-subject factors, which were then used as input for analysis of variance (using the ANOVA function in R) at the group level; significant interactions were decomposed by lower-order tests (phia; https://github.com/heliosdrm/phia). Significance was set at 0.05. Multiple post-hoc tests were Bonferroni-corrected.

\section{Brain imaging}

Structural images $\left(T_{1}\right.$-weighted; $\left.1 \mathrm{~mm}^{3}\right)$ were available for 41 participants (21 patients with anxiety [15 females; mean age $=13.98$ years $]$ and 20 healthy participants [13 females; mean age $=15.32]$ ). The remaining 9 participants were not scanned because of MRI contraindications or refusals. Images were processed using FreeSurfer. ${ }^{52}$ See Appendix 1 for details on acquisition parameters and processing. We conducted analyses using general linear model regressions. Brainstructure measures of interest included cortical thickness and subcortical grey matter volume. We used sex, age and global thickness/intracranial volume as nuisance regressors. ${ }^{53}$

Following our previous research, we carried out analyses using permutation tests in PALM..$^{34,54}$ Whole-brain, vertex-wise inference was based on the cluster extent statistic, with a clusterforming threshold of $p=0.005$. The cluster extent was considered significant if $p<0.05$ after family-wise error (FWE) correction. We also conducted a region-of-interest analysis, testing for replication of our previous finding, which showed that a thinner dIPFC was associated with greater SCR during threat anticipation. ${ }^{34}$ To do so, we manually created a mask including only vertices in the dlPFC cluster found in the previous work. We then performed an analysis identical to the whole-brain analysis (including threshold definition) on anticipatory response but restricted the search space to the mask vertices.

\section{Data analysis}

As detailed in the following sections, our primary analyses focused on psychophysiological response before reversal (blocks 1-4) to extend our previous work. ${ }^{34}$ We first examined cue-based pain-anticipatory response and its brain-structure correlates in healthy youth and youth with anxiety. As noted above, we used sex, age and global thickness/intracranial volume as nuisance regressors. Next, we examined pain experience by testing response to thermal stimulation and its structural correlates. Analyses considering cue-contingency reversal are reported in Appendix 1. To facilitate reproducibility, the imaging processing and analysis pipelines are also provided in Appendix 1.

\section{Pain anticipation}

These analyses examined SCR and brain-structure correlates of cue-based pain-anticipatory responses and their moderation by anxiety.

\section{Psychophysiological response}

We examined SCR in anticipation of painful versus nonpainful stimulation by testing the cue $\times$ group interaction on SCR to cues, using cue (high-pain, low-pain) as a within-subject factor and group (anxiety, healthy) as a between-subjects factor. We used the same model to examine pain-expectancy ratings (averaged across blocks; reported in Appendix 1).

\section{Brain structure}

We examined associations between brain-structure measures and pain-anticipatory SCR by testing the structure (cortical thickness or subcortical grey matter volume) $\times$ group interaction predicting the magnitude of anticipatory SCR. Following our previous work, we indexed pain anticipation by averaging SCR across cues. ${ }^{34}$

\section{Pain experience}

We examined anxiety differences in SCR to pain stimulation and their associations with brain structure, extending previous work. ${ }^{34}$ Given the potential anticipatory effects on pain experience, ${ }^{8,17,40}$ we tested whether anxiety moderated such processes. Subjective pain ratings served as secondary measures.

\section{Psychophysiological response}

We tested the trial type $\times$ group interaction on SCR to heat stimulation; trial type (LL, LM, HM, HH) served as a withinsubject factor, and group (anxiety, healthy) as a betweensubjects factor. Extending previous work, ${ }^{34}$ we tested the main effect of group to identify anxiety differences in overall response to heat. We used the trial type $\times$ group effect to examine whether anticipation of painful versus nonpainful stimulation influenced subsequent response to heat. ${ }^{8,40}$ Of particular interest was the contrast between LM and HM responses, which indexed how anticipation modulated experiences of the same heat intensity. ${ }^{40}$ We tested the same model on subjective pain reports.

Brain structure

We tested the structure $\times$ group interaction on SCR to heat stimulation, averaged across all trials. 


\section{Results}

\section{Participants}

The final sample included 25 healthy participants ( $16 \mathrm{fe}-$ males; mean age [range] $=14.90 \mathrm{yr}[9.30-17.28]$ ) and 25 patients with anxiety (17 females; mean age [range] $=14.06 \mathrm{yr}$ [9.34-17.90]) (Table 1). All 25 patients were medication-free when they completed the task; 23 were medication-naive. The groups did not differ in terms of age, sex or IQ $(p>0.17)$; the anxiety group reported higher current anxiety symptoms $\left(t_{28.31}=9.71, p<0.001\right)$.

\begin{tabular}{lcc}
\multicolumn{4}{l}{ Table 1: Demographic and clinical information } & \\
\hline & $\begin{array}{c}\text { Healthy } \\
(n=25)\end{array}$ & $\begin{array}{c}\text { Anxious } \\
(n=25)\end{array}$ \\
Characteristic & $16(64)$ & $17(68)$ \\
\hline Female, $n(\%)$ & $14.90 \pm 2.01$ & $14.06 \pm 2.16$ \\
Age, yr, mean \pm SD & $108.12 \pm 10.71$ & $112.56 \pm 11.55$ \\
IQ, mean \pm SD* & $6.39 \pm 3.21$ & $28.48 \pm 10.68$ \\
SCARED score, mean \pm SD & - & $21(84)$ \\
Diagnosis, $n(\%)$ & - & $14(56)$ \\
Generalized anxiety disorder & - & $8(32)$ \\
Social anxiety disorder & - & $7(28)$ \\
Specific phobia & - & \\
Separation anxiety disorder & & \\
\hline IQ = intelligence quotient; SCARED = Screen for Child Anxiety Related Emotional \\
Disorders; SD = standard deviation. \\
*Assessed using the Wechsler Abbreviated Scale of Intelligence. \\
†SCARED scores reflect averaged total scores for child and parent reports.
\end{tabular}

\section{Pain anticipation}

\section{Psychophysiological response}

Tests of cue $\times$ group interactions on anticipatory SCR revealed a significant effect $\left(F_{1,1748}=8.61, p=0.003\right)$. Follow-up tests indicated specificity in anxiety-related enhanced anticipatory response: patients with anxiety showed higher SCR to anticipated threat (highly painful stimulation) than their healthy counterparts $(b=0.52, p=0.003)$, and the groups showed comparable anticipatory response to safety (nonpainful stimulation; $b=0.30, p=0.07$; Figure 2). This interaction qualified a significant main effect of cue $\left(F_{1,1748}=76.04\right.$, $p<0.001$ ), indicating higher SCR to high- versus low-pain cues across groups, and a significant main effect of group $\left(F_{1,48}=6.80, p=0.012\right)$, with greater overall anticipatory response in patients with anxiety. Results did not change when sex and age were added as nuisance covariates.

\section{Brain structure}

Analysis of the associations between brain structure and anticipatory SCR did not reveal significant findings that survived the whole-brain threshold. The region-of-interest analysis replicated our previous finding, indicating a significant main effect of cortical thickness on anticipatory SCR in the left dIPFC, such that thinner cortex was associated with greater magnitude of SCR (Figure 3, left; $p_{\mathrm{FWE}}<$ $0.05,17$ vertices; peak: $p<0.001)$. Of note, this cluster also emerged when considering only response to high-pain cues. We observed no other significant effects, including group effects.

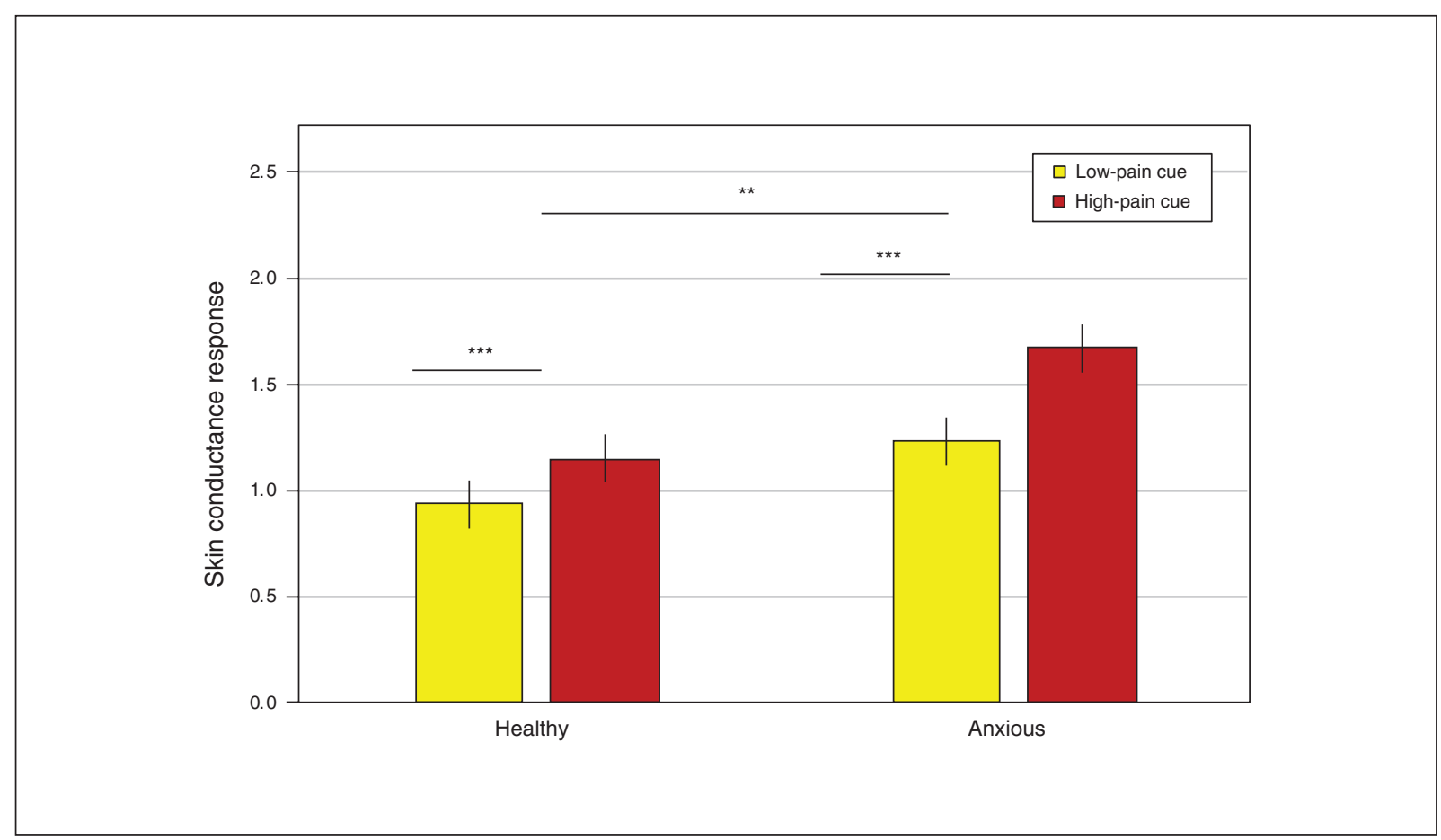

Fig. 2: Psychophysiological response: pain anticipation. Mean skin conductance response (square-root-transformed $\mu S$ ) by cue (low pain, high pain) and group (healthy, anxious). Error bars denote 1 standard error of the mean. ${ }^{* *} p<0.01,{ }^{\star * *} p<0.001$. 
Pain anticipation

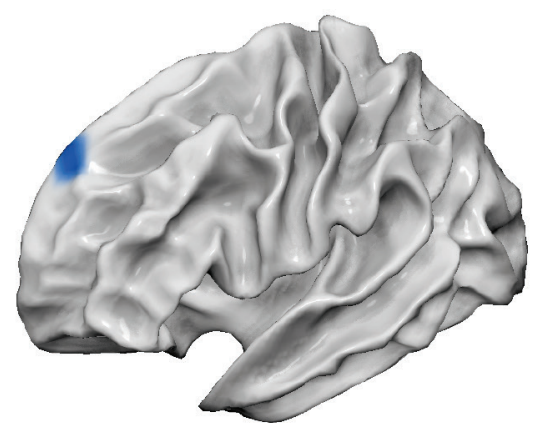

0.05
Pain experience

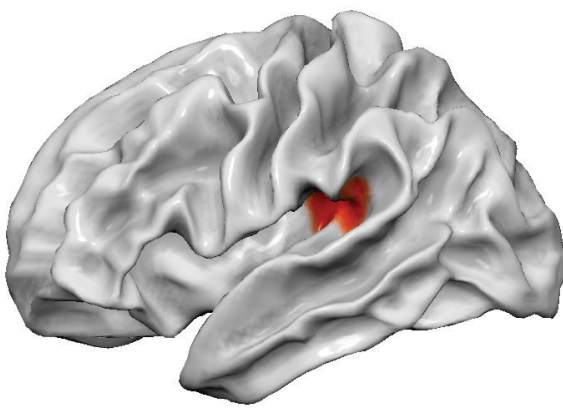

0.05

Positive association

0.001

Fig. 3: Brain structure: pain anticipation and experience. Left: Significant association between left-hemisphere cortical thickness and mean skin conductance response during pain anticipation (averaged response to low and high pain cues). This analysis was conducted on vertices in a region of interest defined by previous work that tested a similar effect. Right: Significant association between lefthemisphere cortical thickness and mean skin conductance response during pain (averaged response across all temperatures delivered). This result was derived from a whole-brain analysis. All results are for analyses using a cluster-forming threshold of $p=0.005$ and a cluster-extent threshold of $p_{\mathrm{FWE}}=0.05$. Colours reflect $p_{\mathrm{FWE}}$ of the cluster.

\section{Pain experience}

\section{Psychophysiological response}

The test of the main effect of group on SCR to heat stimulation yielded a nonsignificant trend for greater response in patients with anxiety $\left(F_{1,48}=4.01, p=0.051\right)$. An exploratory analysis controlling for group difference in anticipatory response (reported above) abolished this trend (group effect after controlling for anticipatory SCR, $p=0.12$; see Appendix 1). A significant main effect of trial type on SCR emerged $\left(F_{3,174}=306.52, p<0.001\right.$; Figure $\left.4 \mathrm{~A}\right)$; follow-up pair-wise tests between all trial types were significant $\left(p_{\mathrm{FWE}}<0.002\right)$. Importantly, SCR in HM trials was greater than in LM trials; this indicated that pain anticipation influenced response to heat, such that response to the same moderately painful heat was greater when painful versus nonpainful heat was expected. However, this effect was not moderated by group (trial type $\times$ group interaction: $p=0.38$; Appendix 1, Figure S3). Results did not change when sex and age were added as nuisance covariates.

A significant main effect of trial type on pain ratings also emerged $\left(F_{3,1744}=1821.09, p<0.001\right.$; Figrue $\left.4 \mathrm{~B}\right)$. Follow-up tests between all trial types were significant, including higher ratings in HM relative to LM trials (all $\left.p_{\text {FWE }}<0.001\right)$. Group moderated the effect of trial type $\left(F_{3,1744}=4.10, p=0.007\right)$, but none of the follow-up tests comparing the groups were significant (all $p_{\mathrm{FWE}}>0.30$ ). We observed no other effects in these analyses. See Appendix 1 for additional exploratory analyses.

\section{Brain structure}

Whole-brain cortical thickness analyses indicated a main effect on SCR to heat pain in the left posterior insula (Figure 3, right; $p_{\mathrm{FWE}}<0.05$, 101 vertices; peak: $p=5.1 \times \mathrm{E}^{-5}$ ), such that thicker cortex was associated with greater SCR magnitude. No other significant effects were observed, including group effects. Additional exploratory analyses testing associations between these factors and pain tolerance indicated the involvement of the somatosensory cortex and are reported in Appendix 1.

\section{Discussion}

This study used painful thermal stimulation to examine links among threat-anticipatory psychophysiological responses, brain structure and pediatric anxiety; 3 key findings emerged. First, youth with anxiety showed enhanced psychophysiological response specifically in anticipation of painful stimulation. Second, the magnitude of the painanticipation response was correlated negatively with dIPFC thickness across all youth. Third, the magnitude of the SCR during pain experience was correlated positively with posterior insula thickness. Together, these findings link brain structure to psychophysiological response during pain anticipation and experience in youth, while highlighting anticipatory response as way to differentiate youth with anxiety from healthy youth.

Our findings indicated that psychophysiological response during threat anticipation differentiated youth with anxiety 
A

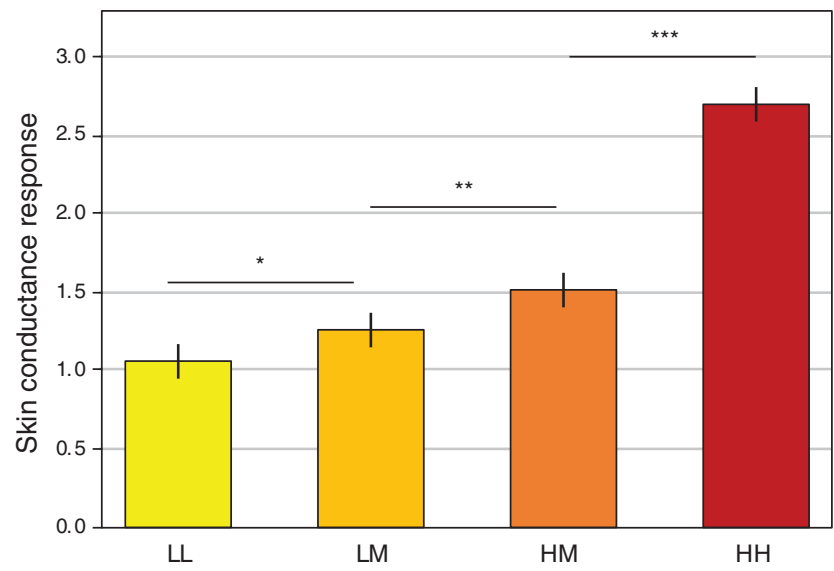

B

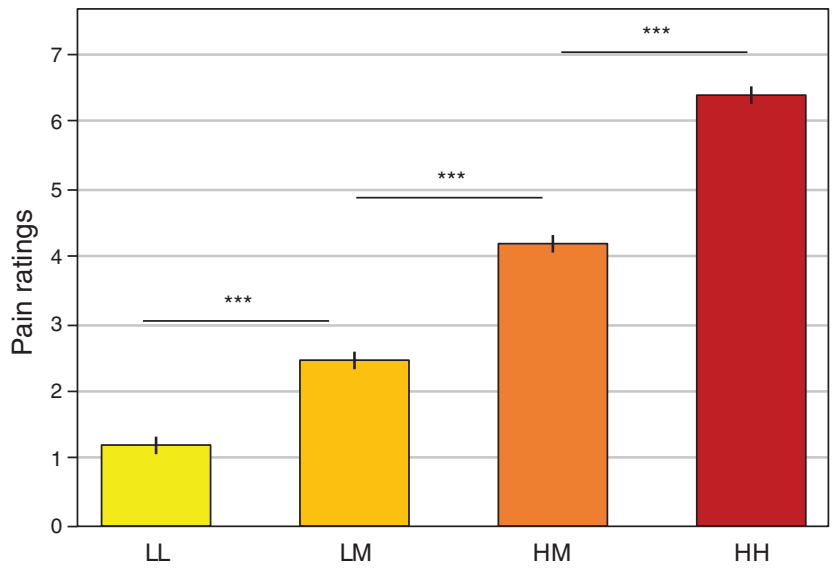

Fig. 4: Response to heat pain. (A) Participants' mean skin conductance response to heat (square-root-transformed $\mu \mathrm{S})$ by trial type (LL, LM, $\mathrm{HM}, \mathrm{HH}$ ). (B) Participants' mean pain ratings of the heat by trial type. Error bars denote one standard error of the mean. Results reflect means across the anxious and healthy groups; see Appendix 1 for means by group. ${ }^{*} p<0.05 ;{ }^{* *} p<0.01$; ${ }^{* *} p<0.001$ (Bonferroni-corrected). $\mathrm{HH}$ = high-pain cue + high-pain temperature; $\mathrm{HM}$ = high-pain cue + medium-pain temperature; LM = low-pain cue + medium-pain temperature; $\mathrm{LL}=$ low-pain cue + low-pain temperature.

from healthy youth. This finding, in a sample including only youth, extends our previous work in an age-diverse sample $e^{34}$ and studied with a less potent reinforcer. Both findings are congruent with evolutionary conceptualizations of psychophysiological responses as preparatory for threat occurrence, and the 2 sets of findings suggest that perturbations in this mechanism characterize pediatric anxiety. ${ }^{1,4,5}$ Excessive threat-anticipatory response is also in line with clinical observations that emphasize exaggerated threat-anticipatory fears in anxiety. ${ }^{11}$ This finding therefore offers a potential mechanistic link between physiological and psychological manifestations of aberrant threat anticipation in anxiety. Future research might continue to examine aspects of this anticipatory phase. ${ }^{5,55}$

Our previous work demonstrated that both probable threat (uninstructed, reinforced conditioned stimulus, CS+) and probable safety (uninstructed, nonreinforced conditioned stimulus, CS-) elicited enhanced anticipatory response in patients with anxiety relative to healthy people. This suggests that threat uncertainty plays a key role in anticipation effects in anxiety, in line with prominent theories. ${ }^{4,13,56}$ The findings of the present study extend previous work by demonstrating that anxiety differences emerge specifically in anticipation of threat. This suggests that threat uncertainty per se may not be a necessary requisite for enhanced threat anticipation in anxiety; ${ }^{39}$ instead, enhanced response could occur to any cue that signals potential danger - whether certain (painful heat cue), probable (CS+) or improbable (CS-) - but not to cues of certain safety (nonpainful heat cue).

The findings also suggest that anxiety differences emerge more prominently in anticipatory response than in response to the actual aversive stimulus. This echoes previous work using different stimulus modalities, ${ }^{34}$ and further focuses the locus of anxiety effects to threat anticipation. This finding was also in line with previous work on face-emotion displays, viewed as intrinsically evocative stimuli. ${ }^{17}$ Experimental paradigms may therefore attempt to distinguish threat anticipation from threat experience, particularly in studies on anxiety. ${ }^{55}$ However, it should be noted that although it was not significant, the magnitude of the anxiety effect on SCR to pain was descriptively larger in the present study than in our previous work. This finding may reflect differences between the tasks. Specifically, in previous work we used a weaker aversive stimulus, which may have diminished individual differences in acute response. Additional work on stimulus characteristics that elicit different anxiety effects could further contribute to our understanding of acute threat response.

In line with our previous work, ${ }^{34}$ we observed a neuroanatomical correlate of anticipatory psychophysiological response. The observed negative association between dIPFC thickness and anticipatory SCR replicated our previous finding ${ }^{34}$ although it should be interpreted with caution because it emerged only in a region-of-interest analysis. The dIPFC function has been previously linked to different instances of threat anticipation such as anticipation of aversive images or electric shock, or to placebo effects. ${ }^{4,26,42,57}$ More broadly, the dIPFC has been hypothesized to play a key regulatory role in emotion regulation. ${ }^{58-60}$ Our work may be seen as complementing such findings, suggesting that thicker dIPFC is associated with better regulation of response during threat anticipation, and informing 
neurodevelopmental theories on emotion regulation and psychopathology. ${ }^{60}$ For example, this finding emerged in a sample of youth and across age in a sample of youth and adults. ${ }^{34}$ Thus, although the dlPFC shows protracted maturation, ${ }^{61}$ regulation of threat-anticipation response is a function that may develop in early age. Nevertheless, longitudinal studies are needed to more conclusively infer whether developmental changes in the association between dlPFC structure and threat anticipation occur. As well, both studies detected this association only in the left dIPFC, suggesting potential laterality effects in the regulation of threat anticipation. Some previous work suggests left laterality in dIPFC function during threat anticipation, ${ }^{42,57}$ although such associations do not arise consistently. ${ }^{20}$ Additional research is required to determine asymmetry in prefrontal cortex structural correlates of anticipatory response.

We also reported novel associations between the thickness of the posterior insula and psychophysiological response to pain. In functional imaging and invasive stimulation studies, the posterior insula has been extensively linked to pain reports and SCR. ${ }^{44,62}$ In the present study, we extended this literature by providing evidence for a link between the posterior insula structure and SCR to pain. Our findings support a neuroanatomical dissociation between pain anticipation and experience. Pain anticipation arguably requires integration of multiple processes to estimate the extent of a potential danger and execute appropriate defensive responses; thus, it may involve the association cortex. ${ }^{4,34,56}$ In contrast, pain experience may require less integrated computation and may be more somatotopically organized; indeed, the insula is hypothesized to show such organization..$^{62}$ The association between insula thickness and response to pain emerged in the right hemisphere. Meta-analyses of neural response to pain suggest right hemispheric dominance for pain processing, with particularly strong laterality effects in the right insula. ${ }^{44,63}$ Relatedly, a right hemisphere dominance in processing emotions has been proposed. ${ }^{64}$ Our findings suggest that this potential functional hemispheric dominance in pain processing may be associated with variation in anatomic structure.

In addition to examining pain anticipation and experience separately, we also tested whether anticipating highly painful versus nonpainful stimulation influenced experiences of moderately painful stimulation. Previous studies demonstrated such effects of expectation influencing subjective pain reports in healthy adults ${ }^{8,40}$ and in healthy youth and youth with anxiety. ${ }^{41}$ Additional work shows that pediatric anxiety moderates the anticipatory effects of uncertainty on neural response to fearful faces. ${ }^{17}$ In the present study, we demonstrated this effect on both pain reports and psychophysiological response: experiencing moderately painful heat elicited greater subjective pain and SCR when preceded by the high-pain cue (HM trials) relative to the low-pain cue (LM trials). Anxiety did not moderate this effect (in line with our previous findings on subjective pain ${ }^{41}$ ), further suggesting that anxiety effects are isolated to threat anticipation but do not affect responses to actual aversive events.

Finally, the anxiety effects demonstrated in the present study emerged in response to anticipated physical stressors.
Pediatric anxiety is often characterized by marked fear of psychological stressors, which are the primary cause of impairment, as observed in separation and social anxiety disorders, for example. The extent to which anticipatory responses to physical and psychological threat share common underlying mechanisms ${ }^{65,66}$ is still unclear. As such, our findings may reflect anticipatory processes that pertain primarily to physical threat, and this possibility suggests the importance of directly contrasting the anticipation of physical and psychological threats in future work.

\section{Limitations}

Several limitations should be considered. First, our sample size was modest, potentially reducing statistical power; this could have particularly affected high-order interactions. Second, we used a cross-sectional design; longitudinal designs would have allowed stronger inferences about developmental and causal associations between threat anticipation and anxiety. Third, brain structure variably maps onto function; ${ }^{28}$ as such, the ability to make strong inferences about the functional roles of identified brain regions was limited. Finally, multiple aspects of data were tested via a number of different analyses; replication of the observed effects is warranted to reduce the risk of spurious effects.

Several strengths mitigated these limitations, and addressed shortcomings in the field of threat induction (for a review, see Lonsdorf and colleagues ${ }^{9}$ ). First, participants were free of medications known to affect psychophysiological response. Second, unlike many studies using visual/auditory aversive stimuli, use of thermal stimulation allows for precise and reliable individual calibration. Moreover, we did not observe habituation to the heat stimulus (in SCR or pain reports) over time.

\section{Conclusion}

Using painful thermal stimulation, we showed that threatanticipatory psychophysiological response differentiated youth with anxiety and healthy youth, and we identified brain-structure correlates of such response. Further, we identified structural correlates of psychophysiological response to heat. Together, these findings highlighted threat anticipation in pediatric anxiety and shed light on the neural circuitry associated with this process.

Acknowledgments: The authors thank the participants and families, as well as the staff of the Intramural Research Program of the National Institute of Mental Health (IRP, NIMH), National Institutes of Health. This research was supported (in part) by the NIMH IRP (ZIAMH002781-15, NCT00018057).

Affiliations: From the Emotion and Development Branch, National Institute of Mental Health, National Institutes of Health, Bethesda, MD (Abend, Bajaj, Harrewijn, Matsumoto, Leibenluft, Pine); the Department of Psychology, University of California Riverside, Riverside, CA (Michalaska); the 3 National Center for Complementary and Integrative Health, National Institutes of Health, Bethesda, MD (Necka, Atlas); and the 1 Department of Psychology, University of Pittsburgh, Pittsburgh, PA (Palacios-Barrios). 


\section{Competing interests: No competing interests declared.}

Content licence: This is an Open Access article distributed in accordance with the terms of the Creative Commons Attribution (CC BY-NC-ND 4.0) licence, which permits use, distribution and reproduction in any medium, provided that the original publication is properly cited, the use is non-commercial (i.e. research or educational use), and no modifications or adaptations are made. See: https://creativecommons.org/licenses/by-nc-nd/4.0/

Contributors: R. Abend, K. Michalska, E. Necka, L. Atlas and D. Pine designed the study. R. Abend, M. Bajaj, A. Harrewijn, C. Matsumoto, E. Necka and E. Palacios-Barrios acquired the data, which R. Abend, M. Bajaj, C. Matsumoto, K. Michalska, E. Necka, E. Lieberluft, L. Atlas and D. Pine analyzed. R. Abend, M. Bajaj, C. Matsumoto, K. Michalska, E. Lieberluft, L. Atlas and D. Pine wrote the article, which all authors reviewed. All authors approved the final version to be published and can certify that no other individuals not listed as authors have made substantial contributions to the paper.

\section{References}

1. Adolphs R. The biology of fear. Curr Biol 2013;23:R79-93.

2. Fanselow MS. The role of learning in threat imminence and defensive behaviors. Curr Opin Behav Sci 2018;24:44-9.

3. LeDoux J. Rethinking the emotional brain. Neuron 2012;73: 653-76.

4. Grupe DW, Nitschke JB. Uncertainty and anticipation in anxiety: an integrated neurobiological and psychological perspective. Nat Rev Neurosci 2013;14:488-501.

5. Hamm AO. Fear, anxiety, and their disorders from the perspective of psychophysiology. Psychophysiology 2020;57:e13474.

6. Dvir M, Horovitz O, Aderka IM, et al. Fear conditioning and extinction in anxious and non-anxious youth: a meta-analysis. Behav Res Ther 2019:120:103431.

7. Merikangas KR, He JP, Burstein M, et al. Lifetime prevalence of mental disorders in U.S. adolescents: results from the National Comorbidity Survey Replication-Adolescent Supplement (NCS-A). J Am Acad Child Adolesc Psychiatry 2010;49:980-9.

8. Palermo S, Benedetti F, Costa T, et al. Pain anticipation: an activation likelihood estimation meta-analysis of brain imaging studies. Hum Brain Mapp 2015;36:1648-61.

9. Lonsdorf TB, Menz MM, Andreatta M, et al. Don't fear 'fear conditioning': methodological considerations for the design and analysis of studies on human fear acquisition, extinction, and return of fear. Neurosci Biobehav Rev 2017;77:247-85.

10. Seifert F, Schuberth N, De Col R, et al. Brain activity during sympathetic response in anticipation and experience of pain. Hum Brain Mapp 2013;34:1768-82.

11. American Psychiatric Association. Diagnostic and statistical manual of mental disorders. 5th ed. Washington (DC): American Psychiatric Publishing; 2013.

12. Duits $\mathrm{P}$, Cath DC, Lissek $\mathrm{S}$, et al. Updated meta-analysis of classical fear conditioning in the anxiety disorders. Depress Anxiety 2015;32:239-53.

13. Grillon C. Startle reactivity and anxiety disorders: aversive conditioning, context, and neurobiology. Biol Psychiatry 2002;52: 958-75.

14. Beesdo K, Knappe S, Pine DS. Anxiety and anxiety disorders in children and adolescents: developmental issues and implications for DSM-V. Psychiatr Clin North Am 2009:32:483-524.

15. Kessler RC, Chiu WT, Demler O, et al. Prevalence, severity, and comorbidity of 12-month DSM-IV disorders in the National Comorbidity Survey Replication. Arch Gen Psychiatry 2005;62:617-27.

16. Craske MG, Hermans D, Vervliet B. State-of-the-art and future directions for extinction as a translational model for fear and anxiety. Philos Trans R Soc Lond B Biol Sci 2018;373: 20170025.

17. Williams LE, Oler JA, Fox AS, et al. Fear of the unknown: uncertain anticipation reveals amygdala alterations in childhood anxiety disorders. Neuropsychopharmacology 2015;40:1428-35.

18. Fullana MA, Harrison BJ, Soriano-Mas C, et al. Neural signatures of human fear conditioning: an updated and extended meta-analysis of fMRI studies. Mol Psychiatry 2016;21:500-8.
19. Carlson JM, Greenberg T, Rubin D, et al. Feeling anxious: anticipatory amygdalo-insular response predicts the feeling of anxious anticipation. Soc Cogn Affect Neurosci 2011;6:74-81.

20. Andrzejewski JA, Greenberg T, Carlson JM. Neural correlates of aversive anticipation: an activation likelihood estimate metaanalysis across multiple sensory modalities. Cogn Affect Behav Neurosci 2019;19:1379-90.

21. Grupe DW, Wielgosz J, Davidson RJ, et al. Neurobiological correlates of distinct post-traumatic stress disorder symptom profiles during threat anticipation in combat veterans. Psychol Med 2016;46:1885-95

22. Wendt J, Low A, Weymar M, et al. Active avoidance and attentive freezing in the face of approaching threat. Neuroimage 2017;158: 196-204.

23. Gu X, Zhou TJ, Anagnostou E, et al. Heightened brain response to pain anticipation in high-functioning adults with autism spectrum disorder. Eur J Neurosci 2018;47:592-601.

24. Ryan KM, Zimmer-Gembeck MJ, Neumann DL, et al. The need for standards in the design of differential fear conditioning and extinction experiments in youth: a systematic review and recommendations for research on anxiety. Behav Res Ther 2019;112: $42-62$.

25. Michalska KJ, Gardiner G, Hughes BL. What neuroscience can tell us about social situations. In: Rauthmann JF, Sherman R, Funder DC, eds. The Oxford handbook of psychological situations. Oxford (UK): Oxford University Press; 2020.

26. Clauss JA, Benningfield MM, Rao U, et al. Altered prefrontal cortex function marks heightened anxiety risk in children. J Am Acad Child Adolesc Psychiatry 2016;55:809-16.

27. Gold AL, Abend R, Britton JC, et al. Age differences in the neural correlates of anxiety disorders: an fMRI study of response to learned threat. Am J Psychiatry 2020;177:454-63.

28. Vazquez-Rodriguez B, Suarez LE, Markello RD, et al. Gradients of structure-function tethering across neocortex. Proc Natl Acad Sci U S A 2019;116:21219-27.

29. Milad MR, Quinn BT, Pitman RK, et al. Thickness of ventromedial prefrontal cortex in humans is correlated with extinction memory. Proc Natl Acad Sci U S A 2005;102:10706-11.

30. Milad MR, Quirk GJ, Pitman RK, et al. A role for the human dorsal anterior cingulate cortex in fear expression. Biol Psychiatry 2007; 62:1191-4.

31. Milad MR, Quirk GJ. Fear extinction as a model for translational neuroscience: ten years of progress. Annu Rev Psychol 2012;63: 129-51.

32. Hartley CA, Fischl B, Phelps EA. Brain structure correlates of individual differences in the acquisition and inhibition of conditioned fear. Cereb Cortex 2011;21:1954-62.

33. Likhtik E, Paz R. Amygdala-prefrontal interactions in (mal)adaptive learning. Trends Neurosci 2015;38:158-66.

34. Abend R, Gold AL, Britton JC, et al. Anticipatory threat responding: relationships with anxiety, development, and brain structure. Biol Psychiatry 2020;87:916-25.

35. Mayberg HS, Liotti M, Brannan SK, et al. Reciprocal limbic-cortical function and negative mood: converging PET findings in depression and normal sadness. Am J Psychiatry 1999;156:675-82.

36. Ochsner KN, Gross JJ. The cognitive control of emotion. Trends Cogn Sci 2005;9:242-9.

37. Elman I, Borsook D. Threat response system: parallel brain processes in pain vis-a-vis fear and anxiety. Front Psychiatry 2018;9:29.

38. Thompson SJ, Bushnell MC. Rodent functional and anatomical imaging of pain. Neurosci Lett 2012;520:131-9.

39. Bennett KP, Dickmann JS, Larson CL. If or when? Uncertainty's role in anxious anticipation. Psychophysiology 2018;55:e13066.

40. Atlas LY, Bolger N, Lindquist MA, et al. Brain mediators of predictive cue effects on perceived pain. J Neurosci 2010;30:12964-77.

41. Michalska KJ, Feldman JS, Abend R, et al. Anticipatory effects on perceived pain: associations with development and anxiety. Psychosom Med 2018;80:853-60.

42. Atlas LY, Wager TD. How expectations shape pain. Neurosci Lett 2012;520:140-8.

43. Peyron R, Fauchon C. The posterior insular-opercular cortex: an access to the brain networks of thermosensory and nociceptive processes? Neurosci Lett 2019;702:34-9.

44. Duerden EG, Albanese MC. Localization of pain-related brain activation: a meta-analysis of neuroimaging data. Hum Brain Mapp 2013;34:109-49. 
45. Kaufman J, Birmaher B, Brent D, et al. Schedule for Affective Disorders and Schizophrenia for School-Age Children-Present and Lifetime Version (K-SADS-PL): initial reliability and validity data. J Am Acad Child Adolesc Psychiatry 1997;36:980-8.

46. Wechsler D. Wechsler abbreviated scale of intelligence. San Antonio (TX): The Psychological Corporation; 1999.

47. Birmaher B, Khetarpal S, Brent D, et al. The screen for child anxiety related emotional disorders (SCARED): scale construction and psychometric characteristics. J Am Acad Child Adolesc Psychiatry 1997;36:545-53.

48. Benedek M, Kaernbach C. Decomposition of skin conductance data by means of nonnegative deconvolution. Psychophysiology 2010;47:647-58.

49. Benedek M, Kaernbach C. A continuous measure of phasic electrodermal activity. J Neurosci Methods 2010;190:80-91.

50. Abend R, Jalon I, Gurevitch G, et al. Modulation of fear extinction processes using transcranial electrical stimulation. Transl Psychiatry 2016;6:e913.

51. Bates D, Mächler M, Bolker B, et al. Fitting linear mixed-effects models using lme4. J Stat Softw 2015;67:1-48.

52. Fischl B, Salat DH, Busa E, et al. Whole brain segmentation: automated labeling of neuroanatomical structures in the human brain. Neuron 2002;33:341-55.

53. Nordenskjold R, Malmberg F, Larsson EM, et al. Intracranial volume normalization methods: considerations when investigating gender differences in regional brain volume. Psychiatry Res 2015;231:227-35.

54. Winkler AM, Ridgway GR, Webster MA, et al. Permutation inference for the general linear model. Neuroimage 2014;92:381-97.

55. Drabant EM, Kuo JR, Ramel W, et al. Experiential, autonomic, and neural responses during threat anticipation vary as a function of threat intensity and neuroticism. Neuroimage 2011;55:401-10.
56. Lissek S, Pine DS, Grillon C. The strong situation: a potential impediment to studying the psychobiology and pharmacology of anxiety disorders. Biol Psychol 2006;72:265-70.

57. Aupperle RL, Allard CB, Grimes EM, et al. Dorsolateral prefrontal cortex activation during emotional anticipation and neuropsychological performance in posttraumatic stress disorder. Arch Gen Psychiatry 2012;69:360-71.

58. Etkin A, Buchel C, Gross JJ. The neural bases of emotion regulation. Nat Rev Neurosci 2015;16:693-700.

59. Phillips ML, Drevets WC, Rauch SL, et al. Neurobiology of emotion perception I: the neural basis of normal emotion perception. Biol Psychiatry 2003;54:504-14.

60. Young KS, Sandman CF, Craske MG. Positive and negative emotion regulation in adolescence: links to anxiety and depression. Brain Sci 2019;9;76.

61. Gogtay N, Giedd JN, Lusk L, et al. Dynamic mapping of human cortical development during childhood through early adulthood. Proc Natl Acad Sci U S A 2004;101:8174-9.

62. Mazzola L, Isnard J, Peyron R, et al. Somatotopic organization of pain responses to direct electrical stimulation of the human insular cortex. Pain 2009;146:99-104.

63. Jensen $\mathrm{KB}$, Regenbogen $\mathrm{C}$, Ohse MC, et al. Brain activations during pain: a neuroimaging meta-analysis of patients with pain and healthy controls. Pain 2016;157:1279-86.

64. Gainotti G. Emotions and the right hemisphere: can new data clarify old models? Neuroscientist 2019;25:258-70.

65. Stein MB, Steckler T, editors. Behavioral neurobiology of anxiety and its treatment. New York: Springer; 2010.

66. Lebowitz ER, Leckman JF, Silverman WK, et al. Cross-generational influences on childhood anxiety disorders: pathways and mechanisms. J Neural Transm (Vienna) 2016;123:1053-67. 